\title{
An Accurate Rate-Equation Description for DFB Lasers and Some Interesting Solutions
}

\author{
Geert Morthier, Member, IEEE
}

\begin{abstract}
Starting from the coupled-wave equations, we have derived an alternative set of rate equations which are valid for most single-section distributed feedback (DFB) lasers. These rate equations are in many respects more useful than the conventional rate equations and have also been used to derive the influence of spatial hole burning on characteristics such as the chirp, the linewidth, or the harmonic distortion. Numerical results are presented for a DFB laser with both facets cleaved, AR-coated, and for a $\lambda / 4$-shifted DFB laser.
\end{abstract}

Index Terms - Distributed feedback lasers, harmonic distortion, modulation.

\section{INTRODUCTION}

A T PRESENT, several longitudinal models for the analysis of distributed feedback (DFB) laser diodes have been reported in the literature [1]-[3]. Such models allow a detailed analysis of effects such as spatial hole burning and are therefore thought to be indispensable in the description of DFB lasers. They are, however, all based on the numerical solution of the static and/or time-dependent, coupled-mode, or other field equations and are therefore for some purposes less attractive than the conventional rate-equation model [4]. Indeed, the simplicity of the rate equations makes it possible to combine them with thermal or electronic models or to implement them in a circuit or system simulator and include, for example, driver electronics or parasitic elements in the analysis [5]. An even bigger advantage of a rate-equation model is that fairly simple analytical expressions for almost all laser characteristics can be derived from it. Such expressions have been and still are very valuable in the design of laser diodes, in the interpretation of measurements, and in the estimation of parameters such as gain and recombination parameters.

However, conventional rate-equation descriptions are not entirely valid or suitable in the analysis of DFB lasers. Conclusions about the design of DFB lasers drawn from such a description are even doubtful. For that reason and because of all the advantages of rate-equation models, there is a need for an alternative, or rather improved, rate-equation description, one which gives an accurate account of the behavior of DFB and Fabry-Perot (FP) lasers. Work in that direction already started a few years ago. Kinoshita [6] has reported a rate-

Manuscript received March 15, 1996; revised July 8, 1996. This work was supported by the European ACTS Project AC065 (BLISS).

The author is with the Department of Information Technology, University of Gent-IMEC, Sint-Pietersnieuwstraat 41, B-9000 Gent, Belgium.

Publisher Item Identifier S 0018-9197(97)00954-8. equation model in which spatial hole burning is included through one additional carrier rate equation. In his model, a number of additional parameters are also introduced and their value must be calculated at each bias level using a static, longitudinal model. This model gives good agreement with longitudinal models but does not include information about chirp or noise. Recently, Schatz [7] reported a lumped smallsignal model that includes longitudinal spatial hole burning. This model is also based on an additional carrier rate equation. It makes use of linearization around a steady-state solution that is obtained from a self-consistent longitudinal simulation. Both models reported in [6] and [7] are put forward as an almost arbitrary generalization of the conventional rate equations in which an effective stimulated emission rate is used. The modification of the photon lifetime is in both models calculated from a static longitudinal model and is therefore a time-independent function of the average photon density. This implies that the time dependence of spatial hole burning is not completely taken into account.

In this paper, we propose an alternative rate-equation model that is derived from the longitudinal equations (in particular the coupled-mode equations and the longitudinal carrier density equations). This derivation leads to a natural averaging with the longitudinal confinement factor as a weighting factor. The final result is a rate equation for the photon density that is exact if the axial profile of the photon density is assumed to be time-independent and that is approximate otherwise. This rate equation resembles [8, Eq. (21)], which has been derived in a totally different manner and which was not further developed into a real rate-equation model. The carrier density is just as in [6] split up in a uniform part and a part that has the axial profile of the photon density. Our approach has the advantage that the extra variables, introduced to include spatial hole burning, only show a weak dependence on the bias level and only need to be calculated once (e.g., at threshold). Assuming these variables constant results in a somewhat less accurate model, but has the great advantage that the resulting model is simple and can be fitted to experimental results. In addition, the use of the longitudinal confinement factor ensures consistency with established approaches such as the use of an effective linewidth enhancement factor [9].

The paper is organized as follows. First, we derive the new rate equations from the coupled-wave equations and the longitudinal carrier rate equations. This derivation is done for the general, large-signal behavior and also takes the noise into account. In Section III, some simple yet accurate solutions 
of the new general rate equations are obtained. Analytical expressions are presented for the linewidth, the chirp, and the second-order harmonic distortion caused by spatial hole burning. They are evaluated and compared with longitudinal solutions in Section IV. To conclude, we summarize the main advantages and properties of our new generalized rate equations.

\section{THEORY}

A description of DFB lasers that is more accurate than the usual rate equations is given by the dynamic coupled-wave equations, and we will therefore derive a rate equation for the photon density from these equations [1]:

$$
\begin{gathered}
\frac{\partial R^{+}}{\partial z}+\frac{1}{v_{g}} \frac{\partial R^{+}}{\partial t}+j \Delta \beta R^{+}=\left(\kappa_{g}+j \kappa_{i}\right) R^{-}+F^{+}(z, t) \\
-\frac{\partial R^{-}}{\partial z}+\frac{1}{v_{g}} \frac{\partial R^{-}}{\partial t}+j \Delta \beta R^{-}=\left(\kappa_{g}^{*}+j \kappa_{i}^{*}\right) R^{+}+F^{-}(z, t) .
\end{gathered}
$$

$R^{ \pm}$are the amplitudes of forward and backward propagating fields, $\kappa_{i}$ is the index coupling, and $\kappa_{g}$ the gain coupling. $F^{+}$and $F^{-}$are uncorrelated Langevin functions with the following second-order moments:

$$
\begin{aligned}
\left\langle F^{+}(z, t) F^{+*}\left(z^{\prime}, t^{\prime}\right)\right\rangle & =\left\langle F^{-}(z, t) F^{-*}\left(z^{\prime}, t^{\prime}\right)\right\rangle \\
& =h \nu \frac{R_{\mathrm{sp}}}{v_{g}} \delta\left(z-z^{\prime}\right) \delta\left(t-t^{\prime}\right)
\end{aligned}
$$

in which $R_{\mathrm{sp}}$ is the spontaneous emission rate and $h \nu$ the photon energy. We now expand the field amplitudes and the complex Bragg deviation in (1) as

$$
\begin{aligned}
& R^{ \pm}(z, t)=R_{0}^{ \pm}(z)+\delta R^{ \pm}(z, t) \\
& \Delta \beta(z, t)=\Delta \beta_{0}(z)+\delta(\Delta \beta(z, t))
\end{aligned}
$$

The field amplitudes $R_{0}^{ \pm}$are the field amplitudes obtained under static operation and $\Delta \beta_{0}(z)$ is the Bragg deviation under static operation. Substitution of the expansions (3) in (1) gives a set of time-dependent coupled-wave equations which can be combined with the static coupled-wave equations (for $R_{0}^{ \pm}$) to yield the following "large-signal" equation for the variation of the Bragg deviation:

$$
\begin{aligned}
\int_{0}^{L} \delta(\Delta \beta)\left[R_{0}^{+} R^{-}+R_{0}^{-} R^{+}\right] d z \\
=\frac{j}{v_{g}} \frac{\partial}{\partial t}\left(\int_{0}^{L}\left(R_{0}^{+} \delta R^{-}+R_{0}^{-} \delta R^{+}\right) d z\right) \\
\quad-j \int_{0}^{L}\left(R_{0}^{-} F^{+}+R_{0}^{+} F^{-}\right) d z .
\end{aligned}
$$

We normalize the fields such that the photon density $S(z)=$ $\left|R^{+}\right|^{2}+\left|R^{-}\right|^{2}$. We also assume that the modulation does not change the field profiles, i.e., that

$$
\delta S(z)=S_{0}(z) f(t), \quad \delta R^{ \pm}=R_{0}^{ \pm}\{\sqrt{1+f(t)}-1\}
$$

with $f$ a real, $z$-independent function of time and $S_{0}(z)$ the static optical power profile. Equation (4) can then be transformed into

$$
\sqrt{1+f} \int_{0}^{L} \delta(\Delta \beta) \Gamma_{z}(z) d z=\frac{j}{v_{g}} \frac{\partial(\sqrt{1+f})}{\partial t}+F_{c}(t)
$$

with the longitudinal confinement factor $\Gamma_{z}(z)$ and $F_{c}$ given by

$$
\begin{aligned}
\Gamma_{z}(z) & =\frac{R_{0}^{+} R_{0}^{-}}{\int_{0}^{L} R_{0}^{+} R_{0}^{-} d z} \\
F_{c}(t) & =\frac{-j}{2 \sqrt{h \nu \nu d v_{g}}} \frac{\int_{0}^{L}\left(R_{0}^{-} F^{+}+R_{0}^{+} F^{-}\right) d z}{\int_{0}^{L} R_{0}^{+} R_{0}^{-} d z} .
\end{aligned}
$$

The Langevin function $F_{c}$ has the following second-order moment:

$$
\begin{aligned}
& \left\langle F_{c}(t) F_{c}^{*}\left(t^{\prime}\right)\right\rangle \\
& =\frac{R_{\mathrm{sp}}}{4 v_{g}^{2} S_{\mathrm{av}} V_{\mathrm{act}}} \delta\left(t-t^{\prime}\right)\left[\frac{\int_{0}^{L}\left[\left|R_{0}^{+}\right|^{2}+\left|R_{0}^{-}\right|^{2}\right] d z}{\left|\int_{0}^{L} R_{0}^{+} R_{0}^{-} d z\right|}\right]^{2} \\
& \quad=\frac{R_{\mathrm{sp}} K_{z}}{v_{g}^{2} S_{\mathrm{av}} V_{\mathrm{act}}} \delta\left(t-t^{\prime}\right)
\end{aligned}
$$

where $K_{z}$ is the longitudinal Petermann factor [10], $S_{\mathbf{a v}}$ the average photon density, and $V_{\text {act }}$ the volume of the active layer. In (6), we can expand $\delta(\Delta \beta)$ as follows:

$$
\begin{aligned}
\delta(\Delta \beta)= & \frac{\delta \omega}{v_{g}}+j \frac{1+j \alpha}{2} \Gamma_{x y} \frac{\partial g}{\partial N} \delta N(z) \\
& -\frac{j}{2} \Gamma_{x y} g_{0} \xi \delta S(z)-\frac{j}{2} \Gamma_{x y} \xi \frac{\partial g}{\partial N} \delta N(z) \delta S(z)
\end{aligned}
$$

with $\alpha$ the linewidth enhancement factor, $\partial g / \partial N$ the differential gain and $\Gamma_{x y}$ the confinement factor of the active layer, and $\xi$ the gain suppression coefficient. We have approximated the variation of the real part of $\Delta \beta$ as a linear function of $\delta \omega$ and $\delta N$. An extension to a nonlinear function would not imply extra complexity, however. Steady-state gain suppression has also been ignored in (9). Substitution of an expression for $\delta N$ and $\delta S$ in (9) and substitution of (9) in (6) results in a set of exact rate equations, one for $f$ and one for $\delta \omega$.

To determine $\delta N$, we can make use of the approximation used in [6] and decompose $N$ and $\delta N$ in a uniform part and a part with the axial variation of $\left(S_{0}(z)-S_{\mathrm{av}}\right)$ as

$$
N=N_{0}(t)+N_{1}(t) F(z) \text { with } F(z)=\frac{S_{0}(z)-S_{\mathrm{av}}}{S_{\mathrm{av}}} .
$$

Substitution of this expansion in (6) and (9) gives the rate equation (11), shown at the bottom of the next page, for $f$ with $G^{\prime}=\Gamma v_{g} \partial g / \partial N, \tau_{d}$ the differential carrier lifetime, and

$$
\chi=\int_{0}^{L}\left(\frac{S_{0}(z)}{S_{\mathrm{av}}}\right) \Gamma_{z}(z) d z
$$

and

$$
\psi=\int_{0}^{L}\left(\frac{S_{0}(z)}{S_{\mathrm{av}}}\right)^{2} \Gamma_{z}(z) d z .
$$

Both quantities $\chi$ and $\psi$ will be complex and fairly independent of the bias level. We will further only use the real parts $\chi^{\prime}$ and $\psi^{\prime}$ and the imaginary parts $\chi^{\prime \prime}$ and $\psi^{\prime \prime}$. 
$\delta N_{0}$ and $\delta N_{1}$ must be determined from the substitution of (10) in the carrier rate equation:

$$
\begin{aligned}
\frac{\partial N}{\partial t}= & \frac{J}{q d}-\frac{N}{\tau(N)}-G(N) S(z)+F_{s}-\frac{2}{h \nu w d} \\
& \times \operatorname{Re}\left\{R^{+^{*}} F^{+}+R^{-^{*}} F^{-}-\left\langle R^{+^{*}} F^{+}+R^{-^{*}} F^{-}\right\rangle\right\} .
\end{aligned}
$$

An expression for the second-order moment of $F_{S}$ is given in [1] but will not be used further on. From (13), one can derive the following equations for $N_{0}$ and $N_{1}$ :

$$
\begin{aligned}
& \frac{d N_{0}}{d t}=\frac{J}{q d}-\frac{N_{0}}{\tau\left(N_{0}\right)}-G\left(N_{0}\right) S_{\mathrm{av}}-\lambda_{1} G^{\prime} N_{1} S_{\mathrm{av}}+F_{N 0} \\
& \frac{d N_{1}}{d t}=-\frac{N_{1}}{\tau_{d}}-G^{\prime} N_{1} S_{\mathrm{av}}-G\left(N_{0}\right) S_{\mathrm{av}}-\lambda_{2} G^{\prime} N_{1} S_{\mathrm{av}}+F_{N 1} .
\end{aligned}
$$

For the sake of simplicity, we have ignored the gain suppression and the spatial variation of the spontaneous carrier recombination. More general equations are given in [6]. The Langevin functions appearing in (13) have been grouped in $F_{N}$ in these equations. $\lambda_{1}, \lambda_{2}, F_{N 0}$ and $F_{N 1}$ are given by

$$
\begin{aligned}
\lambda_{1} & =\frac{1}{L} \int_{0}^{L} F^{2}(z) d z, \quad \lambda_{2}=\frac{\int_{0}^{L} F^{3} d z}{\int_{0}^{L} F^{2} d z} \\
F_{N 0} & =\frac{1}{L} \int_{0}^{L} F_{N} d z, \quad F_{N 1}=\frac{\int_{0}^{L} F_{N} F d z}{\int_{0}^{L} F^{2} d z} .
\end{aligned}
$$

Equations (11) and (14) form our new set of rate equations. Equation (11) can of course be decomposed into an equation for the frequency variation

$$
\begin{aligned}
\delta \omega= & \frac{G^{\prime}}{2}\left[\chi^{\prime \prime} \delta N_{1}+\alpha\left(\delta N_{0}+\delta N_{1}\left(\chi^{\prime}-1\right)\right)\right] \\
& -\frac{\xi G^{\prime}}{2} S_{\mathrm{av}}\left[\chi^{\prime \prime} \delta N_{0}+\left(\psi^{\prime \prime}-\chi^{\prime \prime}\right) \delta N_{1}\right] f \\
& -\frac{\xi G\left(N_{0}\right) S_{\mathrm{av}} f}{2\left(1+G^{\prime} \tau_{d} S_{\mathrm{av}}\right)}\left[\chi^{\prime \prime}-G^{\prime} \tau_{d} S_{\mathrm{av}}\left(\psi^{\prime \prime}-2 \chi^{\prime \prime}\right)\right] \\
& +v_{g} \operatorname{Re}\left\{F_{c}(t)\right\}
\end{aligned}
$$

and an equation for the variation of the average photon density:

$$
\begin{aligned}
\frac{1}{2} \frac{\partial}{\partial t} & \ln (1+f) \\
= & \frac{G^{\prime}}{2}\left[\delta N_{0}+\delta N_{1}\left(\chi^{\prime}-1\right)-\alpha \chi^{\prime \prime} \delta N_{1}\right] \\
& -\frac{\xi G^{\prime} S_{\mathrm{av}} f}{2}\left[\chi^{\prime} \delta N_{0}+\left(\psi^{\prime}-\chi^{\prime}\right) \delta N_{1}\right]-\frac{\xi G\left(N_{0}\right) S_{\mathrm{av}} f}{2} \\
& \times\left\{1+\frac{\chi^{\prime}-1-G^{\prime} \tau_{d} S_{\mathrm{av}}\left(\psi^{\prime}-2 \chi^{\prime}+1\right)}{1+G^{\prime} \tau_{d} S_{\mathrm{av}}}\right\} \\
& -v_{g} \operatorname{Im}\left\{F_{c}\right\} .
\end{aligned}
$$

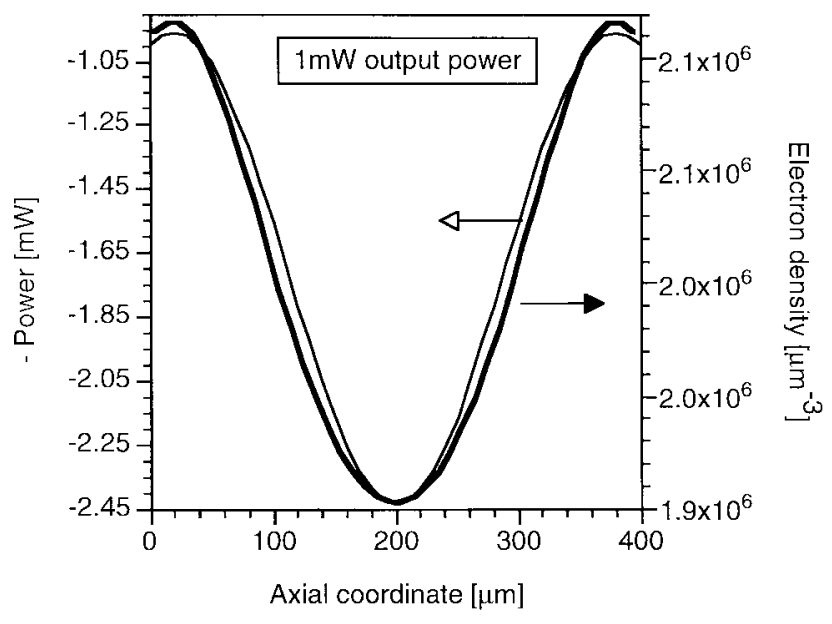

Fig. 1. Longitudinal profile of power and carrier density at 1-mW output power for an AR-coated DFB laser with $\kappa L=3$.

These equations are large-signal equations and are valid as long as the optical power profile resembles that at threshold and as long as the carrier density profile can be expressed accurately as (10). This is the case in most lasers without longitudinal instabilities and without strongly asymmetric power profile. A carrier density expansion which is more accurate over a broader bias level range would be possible in terms of bias dependent base functions, e.g., two orthogonal functions derived from the functions $F_{1}$ and $F_{2}$ with

$$
F_{1}(z)=\frac{1}{1+G^{\prime} \tau_{d} S_{0}(z)} \quad \text { and } \quad F_{2}(z)=\frac{S_{0}(z)}{1+G^{\prime} \tau_{d} S_{0}(z)} .
$$

The expansion (10) has the great advantage that it is independent of the bias level and that it finally leads to a rate-equation system with bias-independent coefficients. The accuracy of the expansion is illustrated for a DFB laser with $\kappa L=3$ and two AR-coated facets in Figs. 1 and 2, which show the longitudinal profiles of power and carrier density at output power levels of 1 and $5 \mathrm{~mW}$. Good agreement between power and carrier density profiles, both being relatively independent of the bias level, is seen.

The parameters $\chi^{\prime}, \chi^{\prime \prime}, \lambda_{1}$, and $\lambda_{2}$ are displayed in Figs. 3, 4 , and 5 as a function of the normalized coupling coefficient $\kappa_{i} L$ for a perfectly AR-coated DFB laser with uniform grating, an AR-coated $\lambda / 4$-shifted DFB laser, and a laser with cleaved facets, respectively. It can be remarked that $\chi^{\prime \prime}=0$ for lasers emitting at the Bragg wavelength, e.g., $\lambda / 4$-shifted lasers. $\chi^{\prime}$ and $\chi^{\prime \prime}$ are the parameters needed in the calculation of the effective linewidth enhancement factor, $\lambda_{1}$ is the variance of

$$
\begin{aligned}
\sqrt{1+f}[\Delta \omega & +\frac{j(1+j \alpha)}{2} G^{\prime}\left[\delta N_{0}+\delta N_{1}(\chi-1)\right]-\frac{j}{2} \xi G^{\prime} S_{\mathrm{av}} f\left[\chi \delta N_{0}+(\psi-\chi) \delta N_{1}\right] \\
& \left.\quad-\frac{j}{2} \xi G\left(N_{0}\right) S_{\mathrm{av}} f\left(1+\frac{\chi-1}{1+G^{\prime} \tau_{d} S_{\mathrm{av}}}-\frac{G^{\prime} \tau_{d} S_{\mathrm{av}}(\psi-2 \chi+1)}{1+G^{\prime} \tau_{d} S_{\mathrm{av}}}\right)\right]=j \frac{\partial(\sqrt{1+f})}{\partial t}+v_{g} F_{c}(t)
\end{aligned}
$$




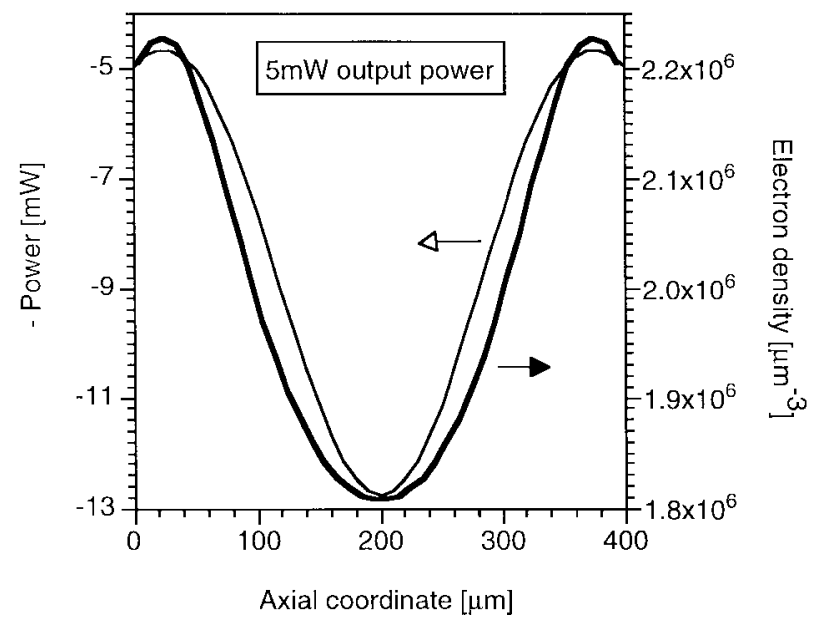

Fig. 2. Longitudinal profile of power and carrier density at 5-mW output power for an AR-coated DFB laser with $\kappa L=3$.

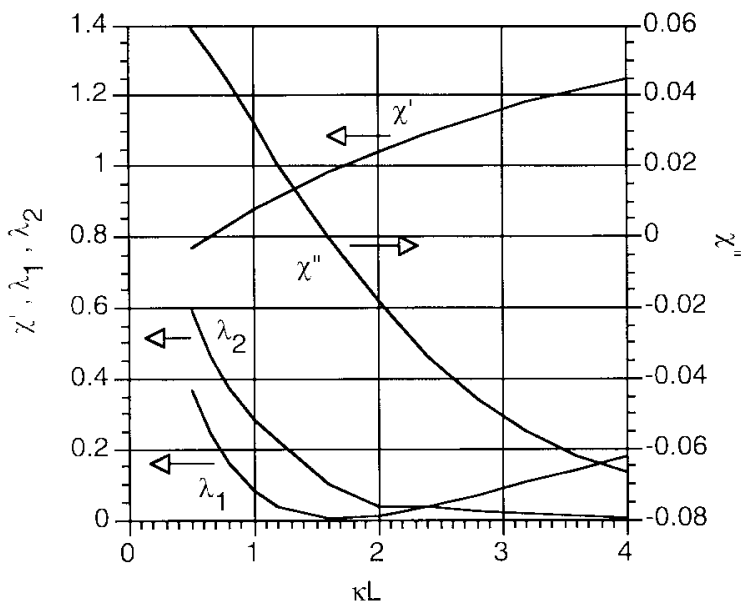

Fig. 3. $\chi, \lambda_{1}$, and $\lambda_{2}$ as a function of $\kappa L$ for a DFB laser with both facets AR-coated (mode with $\lambda>\lambda_{B}$ ).

the longitudinal power distribution, and $\lambda_{2}$ is the ratio of skewness and variance of that distribution.

\section{Simple SMALl-Signal}

\section{SOLUTIONS OF THE RATE EQUATIONS}

Equations (14), (16), and (17) can now be transformed in several ways, e.g., to derive modulation responses, harmonic distortion or noise spectra. Very accurate analytical formulas for these characteristics could be derived from linearization of (14), (16), and (17). Here, we will however simplify the equations further and derive simpler analytical expressions. This illustrates the usefulness and application range of our system of rate equations without requiring extensive numerical simulations. The derived analytical expressions furthermore also shed a light on the influence of spatial hole burning on the chirp and the harmonic distortion.

\section{A. The Linewidth}

We will show here that, for small bias levels, our rate equations lead to the following expression for the linewidth

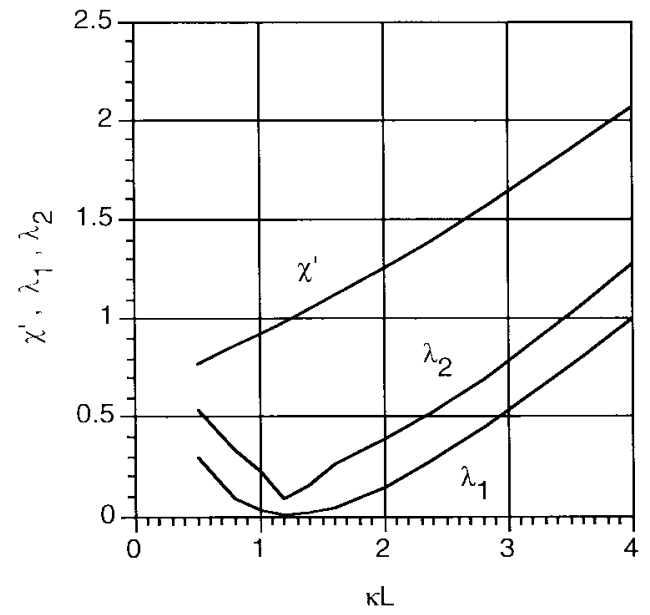

Fig. 4. $\chi, \lambda_{1}$, and $\lambda_{2}$ as a function of $\kappa L$ for a $\lambda / 4$-shifted laser with both facets AR-coated $\left(\chi^{\prime \prime}=0\right)$.

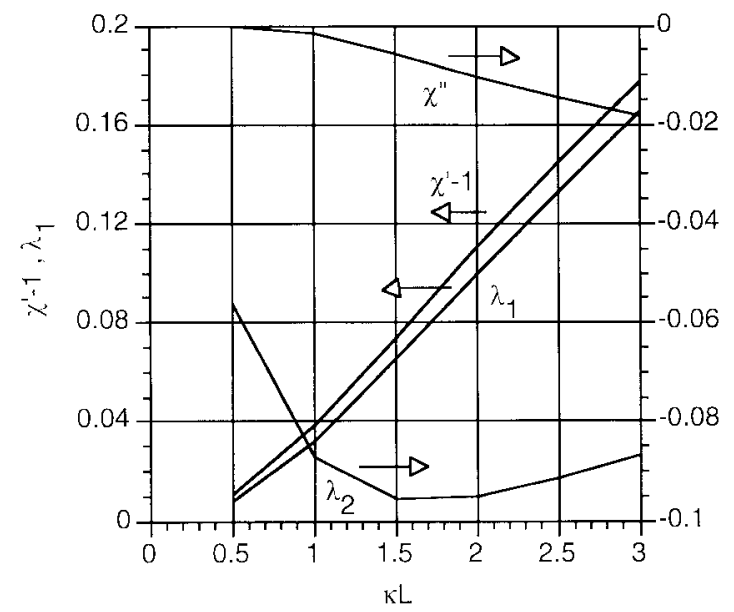

Fig. 5. $\chi, \lambda_{1}$, and $\lambda_{2}$ as a function of $\kappa L$ for a DFB laser with both facets cleaved.

of a DFB laser:

$$
\Delta \nu=\frac{R_{\mathrm{sp}} K_{z}}{4 \pi S_{\mathrm{av}} V_{\mathrm{act}}}\left(1+\alpha_{\mathrm{eff}}^{2}\right)
$$

We assume $G^{\prime} \tau_{d} S_{\text {av }} \ll 1$ and consider only slow fluctuations (i.e., $\partial / \partial t=0$ ). Linearization of (16) and (17) then results in

$$
\begin{aligned}
\delta \omega= & \frac{G^{\prime}}{2}\left[\chi^{\prime \prime} \delta N_{1}+\alpha \delta N_{0}+\alpha \delta N_{1}\left(\chi^{\prime}-1\right)\right]+v_{g} \operatorname{Re}\left\{F_{c}\right\} \\
j \Omega \frac{f}{2}= & \frac{G^{\prime}}{2}\left[\delta N_{0}+\delta N_{1}\left(\chi^{\prime}-1-\alpha \chi^{\prime \prime}\right)\right] \\
& -\frac{\xi G\left(N_{0}\right) S_{\mathrm{av}} f}{2}-v_{g} \operatorname{Im}\left\{F_{c}\right\} .
\end{aligned}
$$

Linearization of the carrier density (14), in which we neglect the Langevin functions and the gain suppression, leads to the following solution for $\delta N_{0}$ and $\delta N_{1}$ :

$$
\delta N_{0}=\delta N_{1}=-G\left(N_{0}\right) S_{\mathrm{av}} \tau_{d} f
$$


Substitution of these expressions in (20), taking into account that $G^{\prime} \tau_{d} \gg \xi$, and elimination of $f$ gives

$$
\begin{aligned}
\delta \omega & =v_{g} \operatorname{Re}\left\{F_{c}\right\}+\frac{\alpha \chi^{\prime}+\chi^{\prime \prime}}{\chi^{\prime}-\alpha \chi^{\prime \prime}} v_{g} \operatorname{Im}\{F\} \\
& =v_{g} \operatorname{Re}\{F\}+\alpha_{\mathrm{eff}} v_{g} \operatorname{Im}\left\{F_{c}\right\} .
\end{aligned}
$$

The second-order moment of $\delta \omega$ and hence the linewidth can be readily derived from (22). The second-order moments of $\operatorname{Re}\left\{F_{c}\right\}$ and $\operatorname{Im}\left\{F_{c}\right\}$ follow from (8) and are equal to one half of the second-order moment of $F_{c}$.

\section{B. The Chirp}

To derive a formula for the chirp, we again linearize (16) and (17) and ignore the Langevin functions. We concentrate on the influence of spatial hole burning and hence neglect the gain suppression again. After Fourier transformation, the small-signal approximations of (16) and (17) then reduce to

$$
\begin{aligned}
\delta \omega & =\frac{G^{\prime}}{2}\left[\chi^{\prime \prime} \delta N_{1}+\alpha \delta N_{0}+\alpha \delta N_{1}\left(\chi^{\prime}-1\right)\right] \\
j \Omega \frac{f}{2} & =\frac{G^{\prime}}{2}\left[\delta N_{0}+\delta N_{1}\left(\chi^{\prime}-1-\alpha \chi^{\prime \prime}\right)\right] .
\end{aligned}
$$

After Fourier transformation, the solutions for $\delta N_{0}(\Omega)$ and $\delta N_{1}(\Omega)$ are found as

$$
\begin{aligned}
& \left(j \Omega+\frac{1}{\tau_{d}}+G^{\prime} S_{\mathrm{av}}\right) \delta N_{0} \\
& =\frac{\delta J}{q d}-G\left(N_{0}\right) S_{\mathrm{av}} f\left\{\frac{1+G^{\prime} \tau_{d} S_{\mathrm{av}}\left(1+\lambda_{2}-\lambda_{1}\right)}{1+G^{\prime} \tau_{d} S_{\mathrm{av}}\left(1+\lambda_{2}\right)}\right\} \\
& \quad-\lambda_{1} G^{\prime} S_{\mathrm{av}} \delta N_{1} \\
& (j \Omega \\
& \left.+\frac{1}{\tau_{d}}+G^{\prime} S_{\mathrm{av}}\left(1+\lambda_{2}\right)\right) \delta N_{1} \\
& =-\frac{G\left(N_{0}\right) S_{\mathrm{av}} f}{1+G^{\prime} \tau_{d} S_{\mathrm{av}}\left(1+\lambda_{2}\right)}-G^{\prime} S_{\mathrm{av}} \delta N_{0} .
\end{aligned}
$$

Substitution of these expressions in the first equation of (23) gives an expression for the FM modulation, while substitution in the last equation of (23) gives an expression for the intensity modulation. If we also neglect the dependence of $\delta N_{1}$ on $\delta N_{0}$ and eliminate $\delta N_{0}$ from the two equations of (23), we find the following expression for the chirp:

$$
\begin{aligned}
\delta \omega=[ & \frac{\alpha}{2} j \Omega-\frac{G^{\prime}}{2} \frac{G\left(N_{0}\right) S_{\mathrm{av}}}{1+G^{\prime}\left(1+\lambda_{2}\right) \tau_{d} S_{\mathrm{av}}} \\
& \left.\times \frac{\chi^{\prime \prime}\left(1+\alpha^{2}\right)}{j \Omega+\frac{1}{\tau_{d}}+G^{\prime}\left(1+\lambda_{2}\right) S_{\mathrm{av}}}\right] f
\end{aligned}
$$

in which $f$ can be considered as the optical modulation depth. Neglecting the dependence of $\delta N_{1}$ on $\delta N_{0}$ is equivalent with assuming that the variation of the average stimulated emission rate is mainly caused by the variation of the photon density and not by the variation of the carrier density. This is always the case above threshold.

The first term in this expression is the dynamic chirp and the second term the chirp caused by spatial hole burning. Equation (25) already implies that for the calculation of the dynamic chirp the material's $\alpha$-factor, not the effective $\alpha$ factor, must be used. This result is in contradiction with previously published analytical formulas [11]. However, we believe that, under the assumption of fixed longitudinal field profiles, our derivation is exact. In fact, also, an expression derived from an exact solution of (24) confirms this.

It is readily seen that the chirp caused by spatial hole burning has a cut-off frequency equal to the inverse carrier lifetime. It also strongly depends on the threshold gain and on $\chi^{\prime \prime}$.

\section{The Harmonic Distortion Caused by Spatial Hole Burning at Low Bias Levels}

We again assume that $G^{\prime} S_{\mathrm{av}} \tau_{d}$ is much smaller than one. We also ignore gain suppression and time dependencies. Equation (17) then reduces to

$$
\delta N_{0}+\delta N_{1}\left(\chi^{\prime}-1-\alpha \chi^{\prime \prime}\right)=0 .
$$

To take into account the nonlinearity of the carrier lifetime $\tau(N)$, we express the carrier recombination $N_{0} / \tau\left(N_{0}\right)$ as

$$
\frac{N_{0}}{\tau\left(N_{0}\right)}=A N_{0}+B N_{0}^{2}+C N_{0}^{3} .
$$

The solution of (26) and of the static carrier rate equations up to second order gives the following result:

$$
\begin{aligned}
\frac{\delta f}{f}= & \frac{\delta_{2} S_{\mathrm{av}}}{\delta S_{\mathrm{av}}} \\
= & \frac{G^{\prime} S_{\mathrm{av}} \tau_{d} f}{2\left(\chi^{\prime}-\alpha \chi^{\prime \prime}\right)} \\
& \times\left\{\lambda_{1}+\left(\chi^{\prime}-1-\alpha \chi^{\prime \prime}\right)\left(1+\lambda_{2}-\chi^{\prime}+\alpha \chi^{\prime \prime}\right)\right\} \\
& -\frac{B+3 C N_{0}}{2\left(\chi^{\prime}-\alpha \chi^{\prime \prime}\right)} G\left(N_{0}\right) S_{\mathrm{av}} \tau_{d}^{2} f\left(\chi^{\prime}-1-\alpha \chi^{\prime \prime}\right)^{2}
\end{aligned}
$$

with $\delta f / f$ the distortion in the average photon density. The distortion in the total output power $P_{\text {out }}$ (from both facets) can be derived from that number by rewriting the equation for $N_{0}$, taking into account that $\left[G\left(N_{0}\right)+\lambda_{1} G^{\prime} N_{1}\right] S_{\mathbf{a v}}=$ $\alpha_{\text {int }} v_{g} S_{\mathrm{av}}+S_{\text {out }}$. The second-order part of that equation

$$
\frac{\delta_{2} N_{0}}{\tau_{d}}+\frac{B+3 C N_{0}}{2}\left(\delta N_{0}\right)^{2}+\alpha_{\text {int }} v_{g} S_{\text {av }} \delta f+\delta_{2} S_{\text {out }}=0
$$

leads to the following expression for the second-order distortion in $P_{\text {out }}$ :

$$
\begin{aligned}
\frac{\delta_{2} P_{\text {out }}}{\delta P_{\text {out }}}= & \frac{G^{\prime} S_{\text {av }} \tau_{d} f}{4 \alpha_{\text {end }}\left(\chi^{\prime}-\alpha \chi^{\prime \prime}\right)} \\
& \times\left\{-\lambda_{1} \alpha_{\text {int }}+\left[\chi^{\prime}-1-\alpha \chi^{\prime \prime}\right]\right. \\
& \left.\times\left[2 \alpha_{\text {end }}\left(1+\lambda_{2}-\lambda_{1}\right)+\alpha_{\text {int }}\left(\chi^{\prime}-\alpha^{\prime} \chi^{\prime \prime}-\lambda_{1}\right)\right]\right\} \\
& -\frac{\left(B+3 C N_{0}\right)}{2\left(\chi^{\prime}-\alpha \chi^{\prime \prime}\right)} G\left(N_{0}\right) S_{\text {av }} \tau_{d}^{2} f\left(\chi^{\prime}-\alpha \chi^{\prime \prime}-1\right)^{2}
\end{aligned}
$$

with $\alpha_{\text {int }}$ the internal loss and $2 \alpha_{\text {end }}$ the total facet loss. We emphasize once more that this expression is only valid at low bias levels. It can be assumed though that this value gives an indication of the spatial hole burning induced distortion at higher power levels also. The last term in the expression is obviously caused by the change in carrier lifetime as a result 
of the change in average gain due to spatial hole burning. It always has a phase $\pi$ and is zero only if there is no variation of the average carrier density or, mathematically, if $\chi^{\prime}-1-\alpha \chi^{\prime \prime}=0$. The first term in the expression can have both a phase zero and a phase $\pi$, depending on the signs of $\left(\chi^{\prime}-1-\alpha \chi^{\prime \prime}\right)$ and $\lambda_{2}$ and on the relative magnitude of internal and facet loss. However, it also has a strong dependence on the differential gain and on the differential carrier lifetime.

For a Fabry-Perot laser, $\chi=1$ and (30) reduces to

$$
\frac{\delta_{2} P_{\text {out }}}{\delta P_{\text {out }}}=-\frac{G^{\prime} S_{\text {av }} \tau_{d} f}{4 \alpha_{\text {end }}} \lambda_{1} \alpha_{\text {int }} .
$$

This is exactly the formula which has been derived in [12].

\section{VALidity OF THE RATE EQUATIONS AND THE ANALYTICAL SOLUTIONS}

We have compared numerical results obtained using the analytical expressions (25) and (30) with results obtained using the longitudinal laser model CLADISS [1]. This gives an indication of both the accuracy of the rate-equation model and the accuracy of the simple analytical expressions. The last are perhaps of more use in the design of laser diodes as they readily show dependencies on all laser and drive parameters and can be easily evaluated.

We first concentrate on the chirp caused by spatial hole burning. At low frequencies, (25) reduces to

$$
\Delta \omega=-\frac{G^{\prime} G\left(N_{0}\right) S_{\mathrm{av}} \tau_{d} f}{2\left[1+G^{\prime}\left(1+\lambda_{2}\right) \tau_{d} S_{\mathrm{av}}\right]^{2}} \chi^{\prime \prime}\left(1+\alpha^{2}\right) .
$$

The contribution from spatial hole burning is proportional with $\chi^{\prime \prime}$, but also increases with the square of $\alpha$, with threshold gain, differential carrier lifetime, and differential gain. For constant optical modulation depth $m=\delta P / P_{0}$, the chirp first increases with bias power, then reaches a maximum $G\left(1+\alpha^{2}\right) \chi^{\prime \prime} m / 8\left(1+\lambda_{2}\right)$ at $S_{\mathrm{av}}=1 /\left[\left(1+\lambda_{2}\right) G^{\prime} \tau_{d}\right]$ and then decreases as $1 / S_{\text {av }}$. There is obviously no spatial hole burning contribution to the chirp in lasers emitting at the Bragg wavelength since $\chi^{\prime \prime}=0$ for such lasers.

The accuracy of (32) has been investigated more in detail for an AR-coated DFB laser with $\kappa L=2$ and $\kappa L=3$. To this end, the results obtained with (32) have been compared with results obtained with CLADISS. The chirp as a function of bias output power for $m=0.2$ obtained with the analytical and the numerical model is shown in Fig. 6 for $\kappa L=2$ and in Fig. 7 for $\kappa L=3$. As can be seen, a very good agreement is obtained up to bias powers of $\pm 1 \mathrm{~mW}$. Above that power level, there is a larger deviation, although the analytical expression (32) still predicts the bias dependence. Fig. 7 shows a better agreement between the analytical formula and the longitudinal calculations than Fig. 6. This indicates that the approximations used in deriving the formula also remain valid for high $\kappa L$ values.

The accuracy of (30) for the spatial hole burning induced second-order harmonic distortion has been investigated for AR-coated $\lambda / 4$-shifted lasers with different $\kappa L$ values. Since (30) is valid only at low bias levels, we have calculated the second-order distortion at a bias power of $0.1 \mathrm{~mW}$ using both

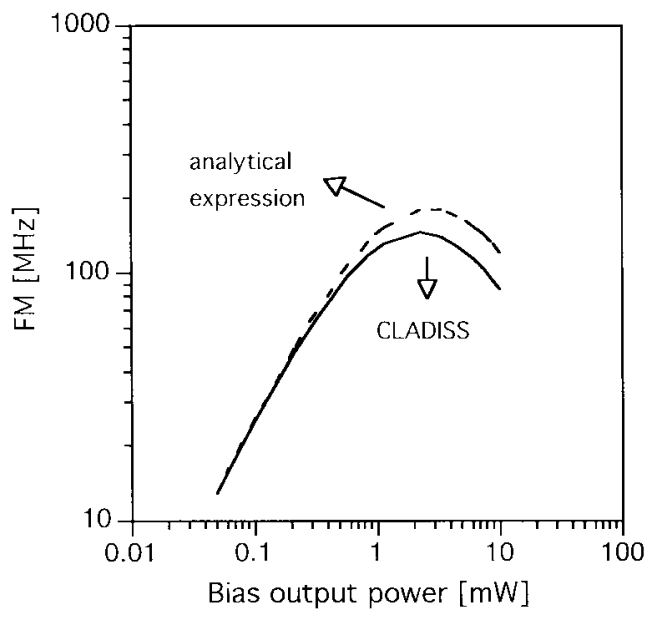

Fig. 6. Low-frequency FM response of an AR-coated (nonphase-shifted) DFB laser with $\kappa L=2$, calculated using (32) and using the longitudinal model CLADISS as a function of the bias output power and for a modulation depth of $20 \%$. Gain suppression has been neglected in the calculations.

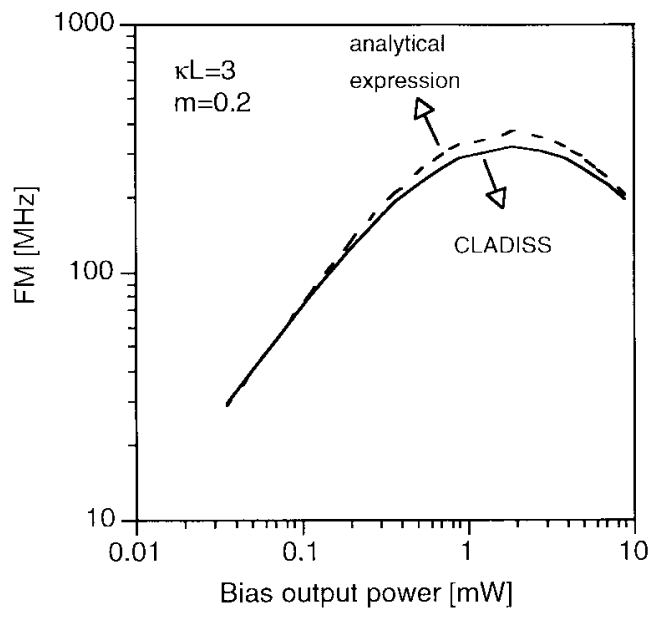

Fig. 7. Low-frequency FM response of an AR-coated (nonphase-shifted) DFB laser with $\kappa L=3$, calculated using (32) and using the longitudinal model CLADISS as a function of the bias output power and for a modulation depth of $20 \%$. Gain suppression has been neglected in the calculations.

CLADISS and the analytical expression. $\mathrm{B}$ and $\mathrm{C}$ have been assumed zero in the calculations. Fig. 8 shows the result as a function of the $\kappa L$ value. Again, a rather good agreement between the analytical and the numerical model has been obtained.

\section{CONCLUSION}

We have presented an alternative set of rate equations for the description of single-section laser diodes. Our rate equations have been derived from the coupled-wave equations and are therefore more useful than the conventional rate equations, in particular in the prediction of spatial hole burning effects. In the derivation, we assumed: 1) that the longitudinal profile of the photon density is independent of the bias level and 2) that the nonuniform part of the electron density is proportional with the nonuniform part of the photon density. Our rate equations are therefore less valid at very high bias levels and for lasers with an unstable power profile. 


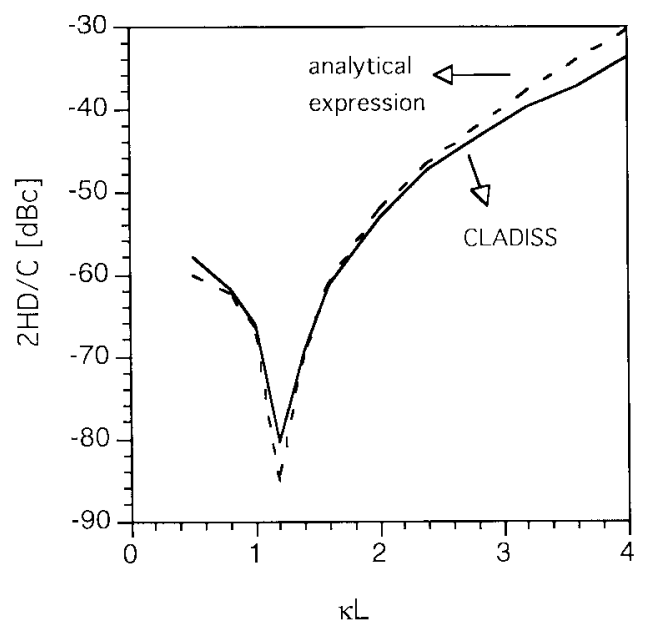

Fig. 8. Second-order harmonic distortion caused by spatial hole burning for AR-coated $\lambda / 4$-shifted DFB lasers at a bias power of $0.1 \mathrm{~mW}$ and for an optical modulation depth of $20 \%$.

These rate equations have been used to derive simple analytical expressions for the linewidth, the chirp, and the harmonic distortion caused by spatial hole burning. Our approach leads naturally to the use of the effective linewidth enhancement factor $\alpha_{\mathrm{eff}}$ and the longitudinal Petermann factor $K_{z}$ in the linewidth formula. The fomula for the chirp includes contributions from spatial hole burning and dynamic effects but is easily extented with a contribution from gain suppression. Important conclusions that follow directly from the formula are the dependence of the dynamic chirp on $\alpha$ and not on $\alpha_{\text {eff }}$ and the dependence of the spatial hole burning induced chirp on threshold gain, differential gain, differential carrier lifetime, and $\alpha^{2}$. The formula derived for the second-order harmonic distortion caused by spatial hole burning finally is valid only at low bias levels, but gives an indication also of the distortion at high bias levels. It readily shows how this distortion depends on the differential gain, the carrier lifetime, the internal and facet loss, and the structural parameters $\chi, \lambda_{1}$, and $\lambda_{2}$.

The parameters $\chi, \lambda_{1}$, and $\lambda_{2}$ introduced in our theory can obviously be considered as additional fingerprints of a DFB laser. Together with the conventional rate-equation parameters and the $K_{z}$ factor, they allow one to describe the smallsignal behavior of a DFB laser, i.e., noise and modulation, completely.

\section{ACKNOWLEDGMENT}

The author wishes to acknowledge R. Baets of the University of Gent-IMEC for useful discussions.

\section{REFERENCES}

[1] P. Vankwikelberge, G. Morthier, and R. Baets, "CLADISS, a longitudinal, multimode model for the analysis of the static, dynamic and stochastic behavior of diode lasers with distributed feedback," IEEE J. Quantum Electron., vol. 26, pp. 1728-1741, Oct. 1990.

[2] A. J. Lowery, A. Keating, and C. N. Murtonen, "Modeling the static and dynamic behavior of quarter-wave shifted DFB lasers," IEEE J. Quantum Electron., vol. 28, pp. 1874-1883, Sept. 1992.

[3] H. Olesen, B. Tromborg, X. Pan, and H. E. Lassen, "Stability and dynamic properties of multi-electrode laser diodes using a Green's function approach," IEEE J. Quantum Electron., vol. 29, Aug. 1993.

[4] G. P. Agrawal and N. K. Dutta, Long-Wavelength Semiconductor Lasers. New York: Van Nostrand Reinhold, 1986.

[5] R. Tucker, "High-speed modulation of semiconductor lasers," J. Lightwave Technol., vol. LT-3, pp. 1180-1192, Dec. 1985.

[6] J. Kinoshita, "Modeling of high-speed DFB lasers considering the spatial holeburning effect using three rate equations," IEEE J. Quantum Electron., vol. 30, pp. 929-938, Apr. 1994.

[7] R. Schatz, "Dynamics of spatial hole burning effects in DFB lasers," IEEE J. Quantum Electron., vol. 31, pp. 1981-1993, 1995.

[8] M.-C. Amann, "Linewidth enhancement in distributed feedback semiconductor lasers," Electron. Lett., vol. 26, pp. 569-571, Apr. 1990.

[9] J. Wang, N. Schunk, and K. Petermann, "Linewidth enhancement for DFB lasers due to longitudinal field dependence in the laser cavity," Electron. Lett., vol. 23, pp. 715-716, July 1987.

[10] W. Huang, X. Li, and T. Makino, "Analytical formulas for modulation responses of semiconductor DFB lasers," IEEE J. Quantum Electron., vol. 31, pp. 842-851, 1995.

[11] G. Morthier, K. David, P. Vankwikelberge, and R. Baets, "Theoretical investigation of the 2nd order harmonic distortion in the AM-response of $1.55 \mu \mathrm{m}$ F-P and DFB lasers," IEEE J. Quantum Electron., vol. 27, pp. 1990-2002, Aug. 1991.

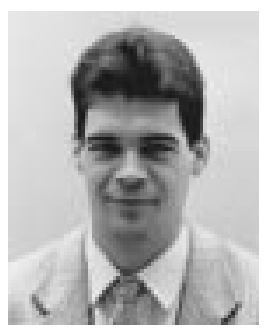

Geert Morthier (M'93) was born in Gent, Belgium, on March 20, 1964. He received the degree in electrical engineering in 1987 and the Ph.D. degree in 1991 from the University of Gent, Gent, Belgium.

Since 1991, he has been a Member of the permanent staff of IMEC, the University of Gent. His main interests are in the modeling and characterization of optoelectronic components. He has authored or coauthored over 40 papers in the field.

Dr. Morthier is a member of the Optical Society of America and the Flemish Engineers Association. 